\title{
The NUMEN project @ LNS: Status and perspectives
}

Cite as: AIP Conference Proceedings 1894, 020004 (2017); https://doi.org/10.1063/1.5007629 Published Online: 16 October 2017

F. Cappuzzello, C. Agodi, L. Acosta, N. Auerbach, J. Bellone, R. Bijker, D. Bonanno, D. Bongiovanni, T. Borello-Lewin, I. Boztosun, V. Branchina, M. P. Bussa, S. Calabrese, L. Calabretta, A. Calanna, D. Carbone, M. Cavallaro, D. Calvo, E. R. Chávez Lomelí, A. Coban, M. Colonna, G. D’Agostino, G. Degeronimo, F. Delaunay, N. Deshmukh, P. N. de Faria, C. Ferraresi, J. L. Ferreira, M. Fisichella, A. Foti, P. Finocchiaro, G. Gallo, U. Garcia, G. Giraudo, V. Greco, A. Hacisalihoglu, J. Kotila, F. Iazzi, R. Introzzi, G. Lanzalone, A. Lavagno, F. La Via, J. A. Lay, H. Lenske, R. Linares, C. Litrico, F. Longhitano, D. Lo Presti, J. Lubian, N. Medina, D. R. Mendes, A. Muoio, J. R. B. Oliveira, A. Pakou, L. Pandola, H. Petrascu, F. Pinna, S. Reito, D. Rifuggiato, M. R. D. Rodrigues, A. D. Russo, G. Russo, G. Santagati, E. Santopinto, O. Sgouros, S. O. Solakcl, G. Souliotis, V. Soukeras, A. Spatafora, D. Torresi, S. Tudisco, R. I. M. Vsevolodovna, R. J. Wheadon, A. Yildirin, and V. Zagatto

\section{ARTICLES YOU MAY BE INTERESTED IN}

The NUMEN project @ LNS: Status and perspectives

AIP Conference Proceedings 2150, 030003 (2019); https://doi.org/10.1063/1.5124592

New experimental campaign of NUMEN project

AIP Conference Proceedings 2150, 030001 (2019); https://doi.org/10.1063/1.5124590

Preface: Matrix Elements for Double Beta Decay Experiments (MEDEX'17)

AIP Conference Proceedings 1894, 010001 (2017); https://doi.org/10.1063/1.5007625
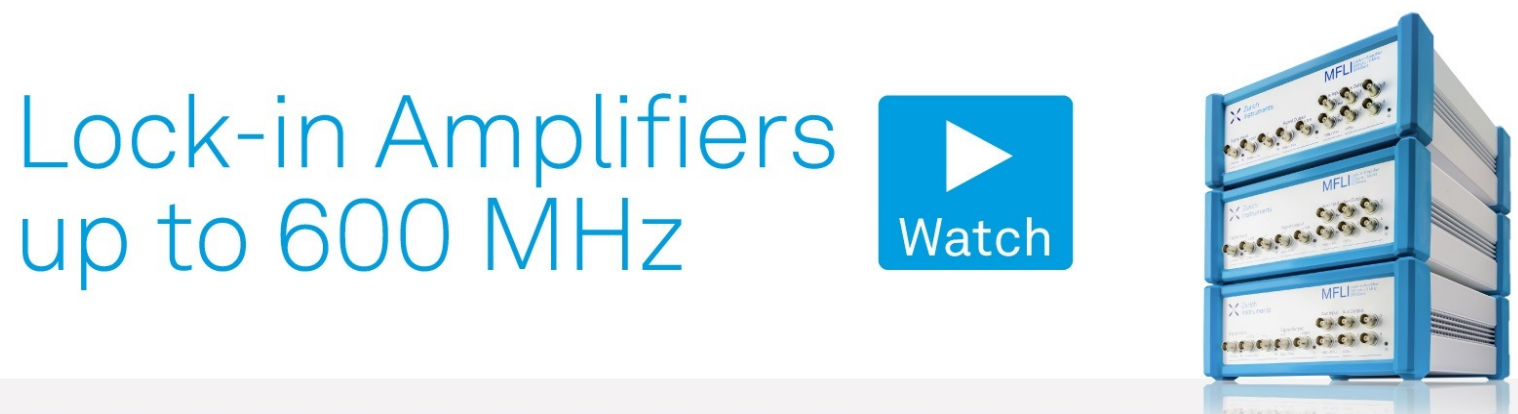


\title{
The NUMEN Project @ LNS: Status and Perspectives
}

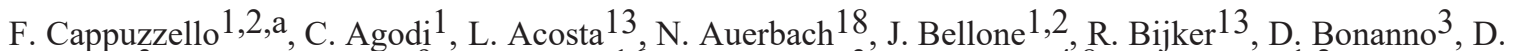
Bongiovanni $^{3}$, T. Borello-Lewin ${ }^{9}$, I. Boztosun ${ }^{16}$, V. Branchina ${ }^{3}$, M. P. Bussa $4^{4,8}{ }^{\text {, S. Calabrese }}{ }^{1,2}$, L. Calabretta ${ }^{1}$, A. Calanna ${ }^{1}$, D. Carbone ${ }^{1}$, M. Cavallaro ${ }^{1}$, D. Calvo ${ }^{4}$, E.R. Chávez Lomelí13, A. Coban ${ }^{16}$, M. Colonna ${ }^{1}$, G. D'Agostino $^{1,2}$, G. Degeronimo ${ }^{21}$, F. Delaunay ${ }^{4,22}$, N. Deshmukh ${ }^{1}$,

P.N. de Faria ${ }^{10}$, C. Ferraresi $^{4}{ }_{\text {J.L. Ferreira }}{ }^{1,10}{ }^{\text {, M. Fisichella }}{ }^{4}$, A. Foti 2,3 , P. Finocchiaro ${ }^{1}$, G. Gallo ${ }^{1,2}$, U. Garcia $^{5}$ G. Giraudo 4 V. Greco 1 , A. Hacisalihoglu 1 , J. Kotila 19 , F. Iazzi 4,6, R. Introzzi 4,6 , G. Lanzalone 1,7, A. Lavagno 4,6 , F. La Via 1 , 14 ${ }^{4}$, J.A. Lay ${ }^{20}$, H. Lenske 15 , R. Linares ${ }^{10}$, G. Litrico ${ }^{1}$, F. Longhitano ${ }^{3}$, D. Lo Presti ${ }^{2}, 3$, J. Lubian $^{10}$, N. Medina ${ }^{9}$, D. R. Mendes ${ }^{10}$, A. Muoio ${ }^{1}$, J.R.B. Oliveira ${ }^{9}$, A. Pakou ${ }^{11}$, L. Pandola ${ }^{1}$, H. Petrascu ${ }^{18}, F_{\dot{5}}$ Pinna $^{4,6}$, S. Reito ${ }^{3}$, D. Rifuggiato ${ }^{1}$, M.R.D. Rodrigues ${ }^{9}$, A. D. Russo ${ }^{1}$, G. Russo ${ }^{2,3}$, G. Santagati ${ }^{1}$, E. Santopinto ${ }^{5}$, O. Sgouros ${ }^{11}$, S.O. Solakc1 ${ }^{16}$, G. Souliotis ${ }^{12}$, V. Soukeras ${ }^{11}$, A. Spatafora ${ }^{1,2}$, D. Torresi ${ }^{1}$, S. Tudisco ${ }^{1}$, R.I.M. Vsevolodovna $^{5}$, R.J. Wheadon ${ }^{4}$, A. Yildirin ${ }^{16}$, V. Zagatto 9

${ }^{1}$ Istituto Nazionale di Fisica Nucleare, Laboratori Nazionali del Sud, Catania, Italy

${ }^{2}$ Dipartimento di Fisica e Astronomia, Università di Catania, Italy

${ }^{3}$ Istituto Nazionale di Fisica Nucleare, Sezione di Catania,

${ }^{4}$ Istituto Nazionale di Fisica Nucleare, Sezione di Torino

${ }^{5}$ Istituto Nazionale di Fisica Nucleare, Sezione di Genova

${ }^{6}$ Politecnico di Torino, Italy

${ }^{7}$ Università degli Studi di Enna "Kore", Enna, Italy

${ }^{8}$ Università di Torino, Torino, Italy

${ }^{9}$ Universidade de Sao Paulo, Brazil

${ }^{10}$ Universidade Federal Fluminense, Niteroi, Brazil

${ }^{11}$ University of Ioannina, Ioannina, Greece

${ }^{12}$ University of Athens and HINP, Athens, Greece

${ }^{13}$ Universidad Nacional Autónoma de México

${ }^{14}$ CNR-IMM, Sezione di Catania, Italy

${ }^{15}$ University of Giessen, Germany

${ }^{16}$ Akdeniz University, Antalya, Turkey

${ }^{17}$ School of Physics and Astronomy Tel Aviv University, Israel

${ }^{18}$ IFIN-HH, Romania

${ }^{19}$ University of Jyväskylä, Jyväskylä, Finland

${ }^{20}$ University of Seville, Spain

${ }^{21}$ Stony Brook University, USA

${ }^{22}$ Université de Caen Normandie and Laboratoire de Physique Corpusculaire de Caen, France

${ }^{a)}$ Corresponding author: cappuzzello@lns.infn.it

\begin{abstract}
The NUMEN project aims at accessing experimentally driven information on Nuclear Matrix Elements (NME) involved in the half-life of the neutrinoless double beta decay $(0 v \beta \beta)$, by high-accuracy measurements of the cross sections of Heavy Ion (HI) induced Double Charge Exchange (DCE) reactions. Particular attention is given to the $\left({ }^{18} \mathrm{O},{ }^{18} \mathrm{Ne}\right)$ and
\end{abstract}


$\left({ }^{20} \mathrm{Ne},{ }^{20} \mathrm{O}\right)$ reactions as tools for $\beta+\beta+$ and $\beta-\beta$ - decays, respectively. First evidence about the possibility to get quantitative information about NME from experiments is found for both kind of reactions. In the experiments, performed at INFN Laboratory Nazionali del Sud (LNS) in Catania, the beams are accelerated by the Superconducting Cyclotron (CS) and the reaction products are detected the MAGNEX magnetic spectrometer. The measured cross sections are challengingly low, limiting the present exploration to few selected isotopes of interest in the context of typically low-yield experimental runs. A major upgrade of the LNS facility is foreseen in order to increase the experimental yield of at least two orders of magnitude, thus making feasible a systematic study of all the cases of interest. Frontiers technologies are going to be developed, to this purpose, for the accelerator and the detection systems. In parallel, advanced theoretical models will be developed in order to extract the nuclear structure information from the measured cross sections.

\section{INTRODUCTION}

The $0 v \beta \beta$ decay of atomic nuclei is a long-searched phenomenon which, besides establishing the Majorana nature of neutrinos, has the potential to shed light on the absolute neutrino mass and hierarchy. A critical aspect is that the associated Nuclear Matrix Elements (NME) must be reliably known. An updated comparison of the results of NME calculations, obtained within various nuclear structure frameworks [1-4], indicates that significant differences are still found, which makes the present situation not satisfactory. In addition, some assumption common to different competing calculations, like the unavoidable truncation of the nuclear many body wave-function, could cause overall systematic uncertainties.

NUMEN [5-6] proposes to use HI-DCE reactions as tools to access quantitative information, relevant for $0 v \beta \beta$ decay NME. These reactions are characterized by the transfer of two charge units, leaving the mass number unchanged, and can proceed by a sequential nucleon transfer mechanism or by meson exchange. Despite $0 v \beta \beta$ decays and HIDCE reactions are mediated by different interactions, they present a number of similarities. Among those, the key aspects are that initial and final nuclear states are the same and the transition operators in both cases present a superposition of isospin, spin-isospin and rank-two tensor components with a relevant available momentum (100 $\mathrm{MeV} / \mathrm{c}$ or so).

\section{ABOUT THE PROJECT}

In a pioneering experiment, performed at the INFN-LNS laboratory, we studied the DCE reaction ${ }^{40} \mathrm{Ca}\left({ }^{18} \mathrm{O},{ }^{18} \mathrm{Ne}\right){ }^{40} \mathrm{Ar}$ at $270 \mathrm{MeV}$, with the aim to measure the cross section at zero degrees [7]. The key elements in the experiment were the high resolution CS beams and the MAGNEX spectrometer, a modern high resolution and large acceptance magnetic system characterized by high resolution in energy, mass and angle [8-10]. The high-order solution of the equation of motion is the key feature of MAGNEX, which guarantees the above mentioned performances and its relevance in the research of heavy-ion physics [11-13]. In the "pilot experiment" we have shown that high resolution and statistically significant experimental data can be measured for DCE processes and that precious information towards NME determination could be at our reach. To move towards nuclei candidates for $0 v \beta \beta$ decay important experimental limits need to be overcome. The challenge is to measure a rare nuclear transition under a very high rate of heavy ions produced by the beam-target interaction. Despite the magnetic fields of the spectrometer filter out the incoming beam and large part of the reaction products, still the events of interest at the focal plane represent less than $10^{-8}$ of the total detected yield. As a consequence, effective rejection properties of unwanted events and radiation hardness are also demanded to the focal plane detectors. The exploration of nuclei of interests for $0 v \beta \beta$ is particularly stimulating as well as challenging. We consider that:

a) The Q-value for DCE reactions on nuclei of interest for $0 v \beta \beta$ is normally more negative (typically about -10 $\mathrm{MeV})$ than in the case of ${ }^{40} \mathrm{Ca}$ explored in ref. [4] $(\mathrm{Q}=-5.9 \mathrm{MeV})$. This could strongly reduce the cross section at very forward angles, especially for $L=0$ transitions.

b) The $\left({ }^{18} \mathrm{O},{ }^{18} \mathrm{Ne}\right)$ reaction is particularly advantageous, due to the large value of both the $\mathrm{B}\left[\mathrm{GT} ;{ }^{18} \mathrm{O}_{\mathrm{gs}}\left(0^{+}\right) \rightarrow\right.$ $\left.{ }^{18} \mathrm{~F}_{\mathrm{gs}}\left(1^{+}\right)\right]$and $\mathrm{B}\left[\mathrm{GT} ;{ }^{18} \mathrm{~F}_{\mathrm{gs}}\left(1^{+}\right) \rightarrow{ }^{18} \mathrm{Ne}_{\mathrm{gs}}\left(0^{+}\right)\right]$strengths and to the concentration of the GT strength in the ${ }^{18} \mathrm{~F}\left(1^{+}\right)$ ground state. However, this reaction is of $\beta^{+} \beta^{+}$kind, while most of the research on $0 v \beta \beta$ is in the opposite side;

c) None of the reactions of $\beta \beta$ kind looks like as favorable as the $\left({ }^{18} \mathrm{O},{ }^{18} \mathrm{Ne}\right)$. For example, the $\left({ }^{18} \mathrm{Ne},{ }^{18} \mathrm{O}\right)$ requires a radioactive beam, which cannot be available with comparable intensity. The proposed $\left({ }^{20} \mathrm{Ne},{ }^{20} \mathrm{O}\right)$ or the $\left({ }^{12} \mathrm{C},{ }^{12} \mathrm{Be}\right)$ have smaller $\mathrm{B}(\mathrm{GT})$, so a sensible reduction of the yield is foreseen in these cases;

d) In some case gas or implanted targets are necessary, e.g. ${ }^{136} \mathrm{Xe}$ or ${ }^{130} \mathrm{Xe}$, which are normally much thinner than solid state ones, with a consequent reduction of the collected yield;

e) In some case the energy resolution we can achieve at the moment (about half $\mathrm{MeV}$ ) is not enough to separate the ground from the excited states in the final nucleus. In these cases, the coincident detection of $\gamma$-rays from the de-excitation of the populated states is mandatory, but at the price of the collected yield. 
As a consequence, the present limits of beam power $(\sim 100 \mathrm{~W})$ for the CS accelerator and acceptable rate for the MAGNEX focal plane detector (few kHz) must be sensibly overcome. For a systematic study of the many "hot" cases of $\beta \beta$ decays an upgraded set-up, able to work with at least two orders of magnitude more luminosity than the present, is thus necessary. This goal can be achieved by a substantial change in the technologies implemented in the beam extraction and in the detection of the ejectiles. For the accelerator the change of the beam extraction technology from electrostatic deflector to a stripper foil is an adequate choice [14]. For the spectrometer the main foreseen upgrades are:

1. The substitution of the present FPD gas tracker, based on multiplication wire technology with a tracker system based on micro patterned gas detector [15];

2. The substitution of the wall of silicon pad stopping detectors with SiC detectors [16] or similar [17];

3. The introduction of an array of detectors for measuring the coincident $\gamma$-rays;

4. The development of suitable front-end and read-out electronics, capable to guarantee a fast read-out of the detector signals, still preserving a high signal to noise ratio [18];

5. Develop a suitable architecture for data acquisition, storage and data treatment, including accurate detector response simulations;

6. The enhancement of the maximum accepted magnetic rigidity, preserving the geometry and field uniformity of the magnetic field [19-22] in order to keep the high-precision of the present trajectory reconstruction;

7. The installation of a beam dump to stop the high power beams, keeping the generated radioactivity under control

In addition, we are developing the technology for suitable nuclear targets to be used in the experiments. Here the challenge is to produce and cool isotopically enriched thin films able to resist to the high power dissipated by the interaction of the intense beams with the target material [23].

Finally, NUMEN is fostering the development of a specific theory program to allow an accurate extraction of nuclear structure information from the measured cross sections. Relying on the use of the DWBA approximation for the cross section, the theory is focused on the development of microscopic models for DCE reactions, employing several approaches (QRPA, shell model, IBM) for inputs connected to nuclear structure quantities. We are also investigating the possible link between the theoretical description of the $0 v \beta \beta$ decay and DCE reactions.

\section{THE PHASES OF NUMEN}

The project is divided into four different phases, covering at least a decade time horizon.

Phase 1: the experiment feasibility

The pilot experiment: the ${ }^{40} \mathrm{Ca}\left({ }^{18} \mathrm{O},{ }^{18} \mathrm{Ne}\right){ }^{40} \mathrm{Ar}$ reaction at $270 \mathrm{MeV}$, with the first experimental data on heavy-ion DCE reactions in a wide range of transferred momenta, was already explored. The results demonstrate the technical feasibility.

Phase2: toward "hot" cases, optimizing experimental conditions and getting first results

The necessary work for the upgrade of both the accelerator and MAGNEX is carried out still preserving the access to the present facility. In the meanwhile, experiments with integrated charge of tens of $\mathrm{mC}$ (about one order of magnitude more than that collected in the pilot experiment) are performed. These are requiring several weeks (4-8 depending on the case) data taking for each reaction, since thin targets (a few $10^{18}$ atoms $/ \mathrm{cm}^{2}$ ) are used in order to achieve enough energy and angular resolution in the energy spectra and angular distributions. The attention is presently focused on a few favorable cases, like for example the ${ }^{116} \mathrm{Sn}\left({ }^{18} \mathrm{O},{ }^{18} \mathrm{Ne}\right){ }^{116} \mathrm{Cd},{ }^{106} \mathrm{Cd}\left({ }^{18} \mathrm{O},{ }^{18} \mathrm{Ne}\right){ }^{106} \mathrm{Pd}$ as $\beta \beta^{++}$like reactions and the ${ }^{116} \mathrm{Cd}\left({ }^{20} \mathrm{Ne},{ }^{20} \mathrm{O}\right){ }^{116} \mathrm{Sn},{ }^{130} \mathrm{Te}\left({ }^{20} \mathrm{Ne},{ }^{20} \mathrm{O}\right){ }^{130} \mathrm{Xe},{ }^{76} \mathrm{Ge}\left({ }^{20} \mathrm{Ne},{ }^{20} \mathrm{O}\right){ }^{76} \mathrm{Se}$ as $\beta \beta$ - like reactions, with the goal to achieve valuable results for them.

Phase3: the facility upgrade

Once all the building block for the upgrade of the accelerator and spectrometer facility will be ready at the LNS a Phase3, connected to the disassembling of the old set-up and re-assembling of the new will start. An estimate of about 18-24 months is considered. 
Phase4: the experimental campaign

The Phase 4 will consist of a series of experimental campaigns at high beam intensities (some p $\mu \mathrm{A}$ ) and long experimental runs. The goal is to reach integrated charges of hundreds of $\mathrm{mC}$ up to $\mathrm{C}$, for the experiments in coincidences, spanning all the variety of candidate isotopes for $0 v \beta \beta$ decay, like: ${ }^{48} \mathrm{Ca},{ }^{76} \mathrm{Ge},{ }^{82} \mathrm{Se},{ }^{96} \mathrm{Zr},{ }^{100} \mathrm{Mo},{ }^{110} \mathrm{Pd}$, ${ }^{124} \mathrm{Sn},{ }^{128} \mathrm{Te},{ }^{130} \mathrm{Te},{ }^{136} \mathrm{Xe},{ }^{148} \mathrm{Nd},{ }^{150} \mathrm{Nd},{ }^{154} \mathrm{Sm},{ }^{160} \mathrm{Gd},{ }^{198} \mathrm{Pt}$.

\section{REFERENCES}

1. J.D. Vergados, H. Ejiri, and F. Simkovic, Reports on Progress in Physics 75, 106301 (2012).

2. P. Vogel, Jour. of Phys. G: Nucl. and Part. Phys. 39, 124002 (2012).

3. J. Engel and J. Menéndez Rep. Prog. Phys. 80, 046301 (2017).

4. S. Dell'Oro, S. Marcocci, M. Viel and F. Vissani, Advances in High Energy Physics 2016, 2162659 (2016).

5. F. Cappuzzello et al. Journal of Physics Conference Series 630, 012018 (2015).

6. C. Agodi, F. Cappuzzello et al. Nuclear and Particle Physics Proceedings 265, 28-30 (2015).

7. F. Cappuzzello, M. Cavallaro et al. Eur. Phys. J. A 51, 145 (2015).

8. F. Cappuzzello, C. Agodi, D. Carbone and M. Cavallaro, Eur. Phys. J. A 52, 167 (2016).

9. A. Cunsolo, F. Cappuzzello, M. Cavallaro, et al., Eur. Phys. Jour.-Special Topics 150, 343-346 (2007).

10. F. Cappuzzello et al., Nucl. Instr. Methods A 763, 314 (2014).

11. F. Cappuzzello, D. Carbone, M. Cavallaro et al., Nature Communications 6, 6743 (2015).

12. D. Pereira et al., Phys. Lett. B 710, 426 (2012).

13. D. Carbone et al. Phys. Rev. C 90, 064621 (2014).

14. L. Calabretta et al. Modern Physics Letters A 32, 17 (2017).

15. M. Cortesi, S. Rost, W. Mittig, et al. Review of Scientific Instruments 88, 013303 (2017).

16. A. Muoio, C. Agodi, D. Bonanno, et al. EPJ Web of Conferences 117, 10006 (2016).

17. D. Carbone, M. Cavallaro et al., Results in Physics 6 (2016) 863-865.

18. G. De Geronimo et al. IEEE Transactions on Nuclear Science 60, 2314-2321 (2013).

19. A. Lazzaro, F. Cappuzzello, A. Cunsolo et al., Nucl. Instr. and Methods A 591, 394 (2008).

20. A. Lazzaro, F. Cappuzzello, A. Cunsolo et al., Nucl. Instr. and Methods A 585, 136 (2008).

21. A. Lazzaro, F. Cappuzzello, A. Cunsolo et al., Nucl. Instr. and Methods A 570, 192 (2007).

22. A. Lazzaro, F. Cappuzzello, A. Cunsolo et al., Nucl. Instr. and Methods A 602, 494 (2009).

23. F. Pinna, S. Bianco, M.Fisichella, F. Iazzi, R. Introzzi, F. Pirri, D. Calvo, C. Agodi, F. Cappuzzello, D. Carbone, M. Cavallaro, Design and fabrication of thin special targets for intense ion beams in NUMEN experiment, submitted to Applied Surface Science, (2017). 